\title{
Analytical substantiation of constructive parameters of rotating field board of plow's body
}

\author{
Vladimir Konovalov ${ }^{1, *}$, Sergey Konovalov ${ }^{1}$, and Victoria Igumnova ${ }^{1}$ \\ ${ }^{1}$ Kuban State Agrarian University named after I.T.Trubilin, 350044, Krasnodar, Russia.
}

\begin{abstract}
The article shows importance of use of dump plowing for formation and maintenance of soil structure and pest control and diseases of cultivated plants. A significant drawback of plowing process is indicated, which is its high energy intensity. To reduce energy consumption, it is proposed to use rotating field boards, which allow replacing sliding friction forces with rolling friction forces. The article presents a description of design of plow's body with rotating field board and notes that without a preliminary analytical study and justification of initial parameters, their use may not be effective. Analytical relations connecting value of radius of rotating field of board, rate of collapse of soil, allowable value of indentation of field board into soil and specific force of cutting resistance of soil that ability to use it for baseline technical parameters. To analyze obtained dependence, the article presents its graphical solution. The authors obtained an expression for determining the value of arm's force of reference reaction from soil to balance a plow's body, in addition, this indicator can be used to adjust a body when working on soils with different physical and mechanical parameters and at different depths.
\end{abstract}

\section{Relevance of research}

In the practice of world agriculture the most common technologies of tillage are intensive and minimal. Intensive or traditional technology involves the use of mould board tillage, which is usually used for ploughshares [6]. Despite the long time of use of plowing process, it does not lose its effectiveness and relevance. In process of plowing, layer's turn is produced with simultaneous undercutting and embedding of weed vegetation, crop residues, fertilizers, as well as lifting from lower layers, washed by precipitation, colloidal particles, which also contributes to formation and maintenance of soil structure at proper level. In addition, the process of plowing is a very effective method of controlling pests and diseases of cultivated plants, which allows us to consider plowing as an element of environmentally safe technology to reduce the use of chemical methods of plant protection $[8,9]$.

\footnotetext{
*Corresponding author: konovalov.v.i@mail.ru
} 
Despite the fact that technological process of plowing for many years of its use has not changed in terms of approach to interaction of working bodies with soil, design of machines for its implementation continues to be constantly improved. Designs of modern and advanced machines combine new technological and technical approaches, for successful implementation of which the necessary condition is minimum energy consumption and high quality of work in implementation of technological process $[1,5]$.

\section{Target setting}

Plow share tillage is a highly energy-intensive process. During operation of plow is positive, which occurs as a result of direct performance of work, and harmful resistance, which arises due to movement of plow, friction forces, etc. The proportion of required energy to overcome friction forces of working bodies of plow on soil reaches high values, including friction of field boards on furrow's wall, it is even more significantly manifested when working on wet soils $[5,11]$.

At present time for reducing energy intensity of moldboard tillage process are increasingly began to apply the effect to reduce friction of working bodies of soil through use of various polymers with lower coefficients of friction, or replace forces of sliding friction, force of rolling friction.

On the basis of specified methods there were designed ploughshares, mould boards and field boards with application of rolling elements. This direction of improvement of designs is very promising, however their application can be not effective without preliminary analytical research and justification of initial parameters. Structurally, the easiest way to reduce proportion of harmful resistance is achieved through use of rotating cylindrical or conical field boards, which can be located on plow body both in series and in tiers, and also have various elements to create vibrations on them. Such technical solutions have a number of advantages over their analogues $[4,10]$.

\section{Analysis of achievements and publications}

One of the promising designs of plow body (Fig. 1) [6] consists of rack 1, on which is installed with blade 2 and ploughshare 3, and field board 5 mounted on two supports 4, made in form of rotating cylindrical drum with disk knives 6 and cylindrical spacers 7 . Supports 4 to rack 1 are attached to 8 through hinges, and between the support and the blade is mounted spring 9. By swapping and adding cylindrical spacers and circular knives, you can change height of installation and intensity of soil crumbling. Field board is attached with help of hardware or hooks to supports 4 through holes 11 , which allows creating different moments of resistance relative to rack 1 of body and, accordingly, working on soils with different physical and mechanical characteristics. 

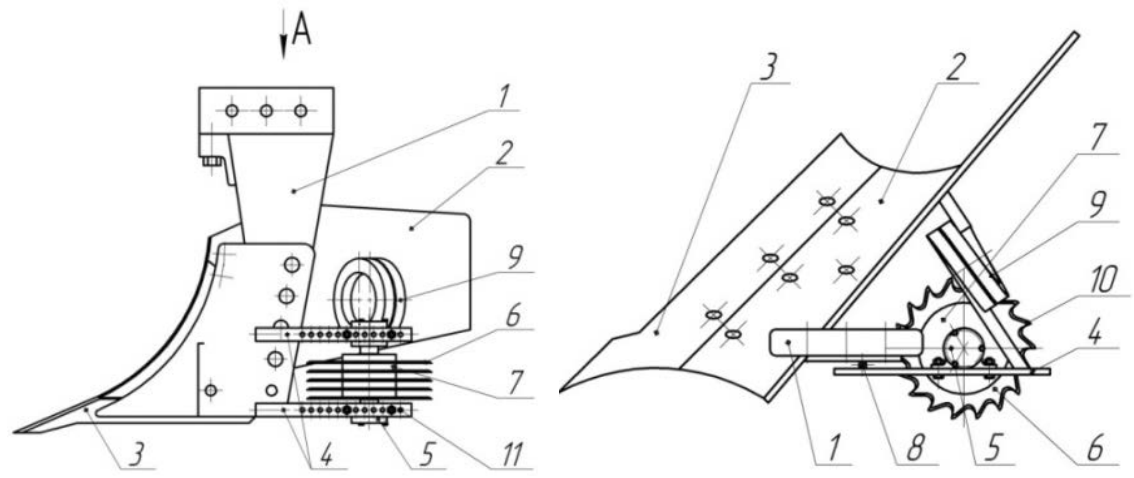

Fig. 1. General view of plow's body.

1 - bar; 2 - mouldboard; 3 - ploughshare; 4 - bearing; 5 - field board; 6 - disk knife; 7 - cylindric plate; 8 - bearing; 9 - spring; 10 - cutting flange; 11 - control hole

The proposed body of ploughshare despite the combination of different constructive and technological approaches is very difficult to manufacture and adjust. Therefore, at initial stage, we will consider supports with rigid fastening to rack, field board in form of rotating solid cylinder and operational process without vibration.

\section{Presentation of main material of research}

A necessary condition for sustained process of body's operation is balance of forces and reactions. [2]. Forces of normal reactions of field boards is due to their interaction with furrow's wall. Then it can be expressed by specific resistance of soil to crumpling, or supporting capacity of soil, which will be equal to [2]:

$$
T_{A}=\frac{P}{S},
$$

where $T_{A}-$ specific resistance of soil to crumpling, $\mathrm{H} / \mathrm{cm}^{2}$;

$P$ - force of soil resistance to crumpling, $\mathrm{H}$;

$S$ - area of contact of supporting surfaces, $\mathrm{cm}^{2}$.

It is obvious that during movement of ploughshare on field, physical and mechanical properties of soil will differ from each other, which will have an even greater impact on its moisture. Then force of soil resistance to crumpling cannot be taken as a constant value and for further research it will replace on value of permissible indentation of field board into soil, which will be equal to $h=5-10 \mathrm{~mm}$ [2]. Figure 2 shows the scheme of interaction of rotating field board of radius $R$ with soil, which shows that the contact line is an arc, indicating about non-uniformity of distribution of forces along it. 


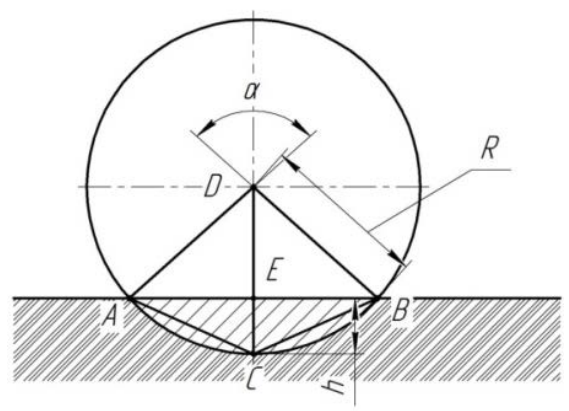

Fig. 2. Scheme of interaction of rotating field board with soil.

In this case, specific resistance of soil to crumpling can be characterized by coefficient of volumetric soil crumple, determined by the formula [2]:

$$
g=\frac{P}{V}
$$

where $g$ is coefficient of bulk soil crumpling, N/cm ${ }^{3}$, for plowed field $g=1-2 \mathrm{~N} / \mathrm{cm}^{3}$, for stubble and fallows $g=5-10 \mathrm{~N} / \mathrm{cm}^{3}$;

$V$ - volume of washed soil, $\mathrm{cm}^{3}$.

Force of resistance to soil cutting by plow's body is decomposed into two components (Fig. 3), while one component pushes field board into wall of furrow, and other component is directed to opposite side of movement of body. Magnitude of indentation of plow's body of field board into soil of the furrow's wall depends on angle between ploughshare and furrow's wall, as well as coefficient of friction of soil on surface of body. The force of indentation will be determined by the formula:

$$
F_{2}=F \cos \left(\gamma_{0}+\varphi\right)
$$

where $\mathrm{F}_{2}$ is force pressing the field board into furrow's wall, $\mathrm{H}$;

$\gamma_{0}$ is angle of ploughshare to bottom of furrow, deg;

$\varphi$ - angle of friction of soil on material of ploughshare, deg;

F- soil cutting resistance force, $\mathrm{N}$.

There are forces of resistance to crumpling which will be distributed in direction of arc of $A S B$ (Fig. 2) at introduction of field board in soil, in which a chord value is determined by length of segment $A B$. The value of chord length can be easily calculated by the Huygens formula, which has the form [3]:

$$
L_{\text {дуги }}=2 C B+\frac{2 C B-A B}{3},
$$




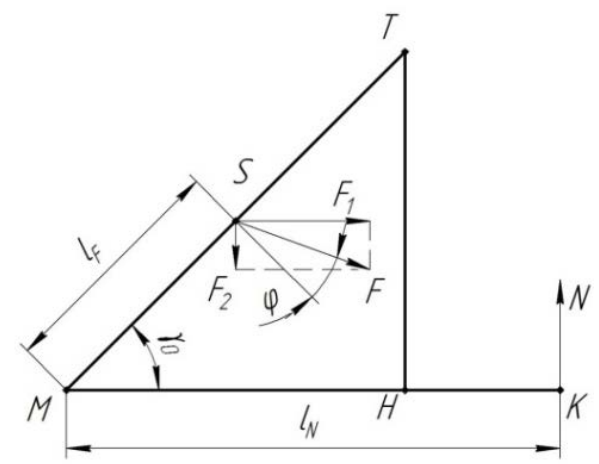

Fig. 3. Scheme of distribution of soil resistance forces to cutting

Since triangle $S E$ is rectangular, value of segment $S V$ will be:

$$
C B=\sqrt{E C^{2}+E B^{2}},
$$

Specify $A E=E B=l, D B=A D=R$, so $A B=2 l, E C=R-h$. From triangle $D E B$ we obtain:

$$
R^{2}=(R-h)^{2}+l^{2}
$$

After transformations we obtain:

$$
l=\sqrt{2 R h-h^{2}},
$$

After substitution of expressions (7) and (5) in the expression (4) and transformations we obtain:

$$
L_{\text {arc }}=\frac{8 \sqrt{2 R h}-2 \sqrt{h(2 R-h)}}{3},
$$

where $L_{\text {arc }}$ - length of arc of supporting surface, $\mathrm{cm}$.

So, area of supporting surface will be:

$$
S_{\text {sup }}=\frac{8 \sqrt{2 R h}-2 \sqrt{h(2 R-h)}}{3} l_{\text {drum }}
$$

where $S_{s u p}$ - area of supporting surface of rotating field board, $\mathrm{cm}^{2}$.

$l_{\text {drum }}$ - length of forming cylinder of rotating field board contacting with soil, $\mathrm{cm}$.

Soil displaced by field board (Fig. 2) represents the segment of $A C B$, area of which is determined by the expression [3]:

$$
S_{s e g}=R^{2} \arccos \left(\frac{R-h}{R}\right)-(R-h) \sqrt{h(2 R-h)},
$$

Then volume of displaced soil will be:

$$
V_{\text {soil }}=\left(R^{2} \arccos \left(\frac{R-h}{R}\right)-(R-h) \sqrt{h(2 R-h)}\right) l_{d t u m},
$$

where $V_{\text {soil }}$ - volume of displaced soil of field board, $\mathrm{cm}^{3}$. 
Next, we determine specific force of pressure of field board on soil, which, according to the expression (9) will be equal to:

$$
P_{\text {drum }}=\frac{3 F_{2}}{\left(8 \sqrt{2 R h}-2 \sqrt{h(2 R-h)) l_{d r u m}}\right.},
$$

Then, taking into account the expression (2) and (11) and a number of transformations, coefficient of volume crumpling of soil will be equal to:

$$
g=\frac{3 F_{2}}{l_{\text {drum }}^{2} \sqrt{h(2 R-h)}\left(R^{2} \arccos \left(\frac{R-h}{R}\right)-(R-h)(8 \sqrt{2 R h}-2)\right.},
$$

Obtained dependence (13) relates value of radius of rotating field board $R$, soil crumpling coefficient $g$, permissible value of field board pressing into soil $h$ and specific force of soil cutting resistance $F$. This dependence is shown in figure 4 graphically.

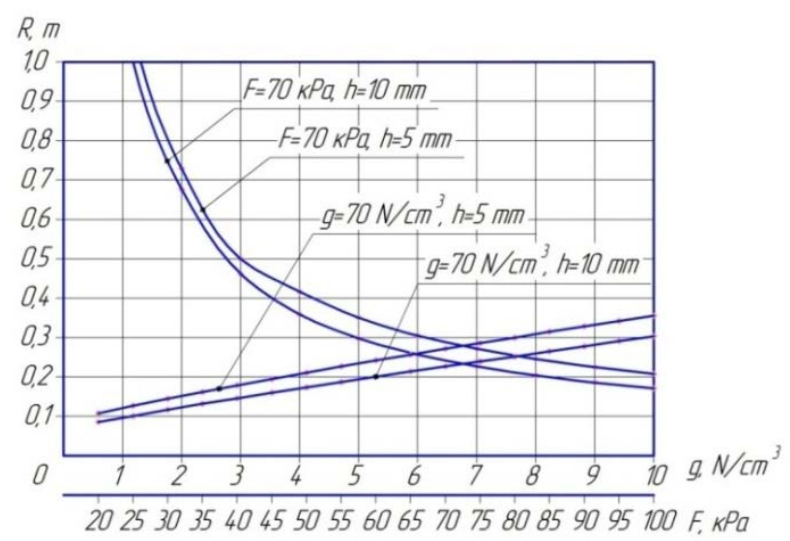

width of grip $b=0,35 \mathrm{~m}$; depth of plowing $a=0,24 \mathrm{~m}$; angle of installation of ploughshare to bottom of furrow $\gamma_{0}=45^{\circ}$; angle of friction of soil $\varphi=25^{\circ}$; length of forming cylinder of field board contacting to soil $1_{\text {drum }}=0,2 \mathrm{~m}$

Fig. 4. Diagram of dependence of radius of rotating field board on soil from crumpling coefficient $g$ and specific force of soil of cutting resistance $F$, at different values of permissible value of indentation $h$

After analyzing graphical dependence, it can be concluded that increase in volume of crumpling coefficient $g$ leads to sharp increase in radius $R$ of rotating field board. On the other hand, when changing permissible soil crumpling $h$ from $5 \mathrm{~mm}$ to $10 \mathrm{~m}$ leads to increase in radius $R$ of rotating field board in a very small range. As soil resistance to cutting $F$ increases, radius $R$ of rotating field board increases, and the change occurs on linear law.

According to presented graphical information the body of plow in soil with resistance of cutting equal to $70 \mathrm{kPa}$ and coefficient of volumetric crumpling $7 \mathrm{~N} / \mathrm{cm}^{3}$ and allowable crumpling of soil $h=5 \mathrm{~mm}$, radius $R$ of rotating field board will be $0,270 \mathrm{~m}$. Using the received value of radius of rotating field board during manufacture of plow's body is not possible, due to the fact that width of furrows for frames are typically 30 to $40 \mathrm{~cm}$. It follows from this that in manufacturing the plow's body with rotating field board to work in these conditions, it is necessary to use a large number of field boards, for example, two, which will reduce the value of their radius. 
In addition to permissible value of pressing a rotating field board into soil, it is necessary to ensure horizontal stability of an istallation [13] for highly effective operation. To do this, it is necessary to make equal moments arising from forces on a ploughshare due to cutting resistance and on contact surface of rotating field board due to reaction forces of soil support. We take a wiper of ploughshare for the center of rotation (Fig. 3). Then equilibrium of condition relative to a wiper of ploughshare will be:

$$
F l_{F}=g V_{\text {soil }} l_{N},
$$

where $l_{F}$ and $l_{N}-$ arm of action of $F$ and $N$ respectively.

From triangle $M T H$ :

$$
l_{F}=\frac{T H}{2 \sin \gamma_{0}},
$$

As far as the side of $T H$ is width of grip of a body, substituting expressions (15) in (14) we obtain:

$$
l_{N}=\frac{F b}{2 g V_{\text {soil }} \sin \gamma_{0}},
$$

After analyzing the expression 16, it can be concluded that increase in specific force of cutting resistance leads to increase in an arm according to linear dependence, and increase in coefficient of volumetric soil crumpling to its decrease in curvilinear dependence.

\section{Conclusions}

To reduce energy intensity of plowing process, one of the effective ways is to replace the standard field board with a rotating one, which will reduce the amount of friction forces. The choice of constructive and technological parameters of cylindrical drum depends on a number of operational and soil-climatic conditions. Theoretical relationships have been derived showing the relationship of technological and operational parameters and soil and climatic conditions, and also allow us to determine radius of cylindrical drum of field board and size of arm of support reactions force on the part of soil to balance a plough's body.

\section{References}

1. S. V. Belousov, Patent search of constructions of supplying soil processing with layer rotation. Method of searching. The proposed technological decision, Polythematic network electronic scientific journal of Kuban State Agrarian University, v. 108. pp. 409-443 (2015)

2. E. S. Bosoy, I. I.Smirnov, O. V. Vernyaev, E. G. Sultan-shakh, Theory, construction and calculation of agricultural machines (Moscow, "Mechanical engineering", 1978)

3. M. Ya. Vygodsky, Guide to Higher Mathematics (Moscow: AST: Astrel, 2006)

4. Yu. K. Kastidi, D. A. Krepyshev, Economic efficiency of Krasnodar Territory commodity producers supply with agricultural machinery, Proceedings of Kuban State Agrarian University, v. 28. pp. 39-42 (2011)

5. G. Maslov, A. S. Serganov, Innovative technology in energy-saving technologies of field crops, Proceedings of Kuban State Agrarian University, v. 71, pp. 110-117 (2018) 
6. V. I. Konovalov, S. I. Konovalov, O. A. Sterligov, Plough's body, Patent 173221 of the RF, MPK A01V 17/00, applicant and patent holder. Federal State Budgetary Educational Institution "Kuban State Agrarian University named after I.T.Trubilin», № 2017106235, order 22.02.2017, published 16.08.2017, Bul, v 23. pp. 5

7. P. N. Rybalkin P. N. [et al.], New adaptive energy-and soil-saving technologies of winter wheat and maize cultivation in Krasnodar Territory, (Krasnodar: Russian Academy of Agricultural Sciences, Krasnodar Research Institute of Agriculture. P. P. Lukyanenko, 2002)

8. P. N. Rybalkin P. N. [et al.], Agronomic requirements for main technological operations with adaptive technologies of winter cereals and maize cultivation and new technical means for their implementation in Krasnodar Territory, (Krasnodar: Department of agriculture and food of Krasnodar Territory, Russian Academy of Agricultural Sciences, Krasnodar Research Institute of Agriculture. P. P. Lukyanenko, 2001)

9. V.A. Romanenko [et al.], Agricultural machines (device, operation and basic adjustments), (Krasnodar, KubSAU, 2014)

10. A. P. Sokolova, Yu. K. Kastidi, G. F. Bershitskaya, M. E. Trubilin, Impact of machinery supply on economic performance of crop production, Rural mechanic, v. 2, pp. 22-23 (2015)

11. E. I. Trubilin, K. A. Sokht, V.I. Konovalov, V.V.Kravchenko, Embedding ability of disk harrows and the technological reliability of disk harrows and ploughs, Equipment and machinery for rural areas, v. 6, pp. 31-34 (2013)

12. E. I. Trubilin, K. A. Sokht, V. I. Konovalov, Improving the technological reliability of disk harrows and ploug, Equipment and machinery for rural areas, v. 6, pp. 12-15 (2013)

13. E.I. Trubilin, K.A. Sokht, V.I. Konovalov, Balance of disk harrows and ploughs in horizontal plane, Proceedings of Kuban State Agrarian University, v. 40. pp. 166-169 (2013) 